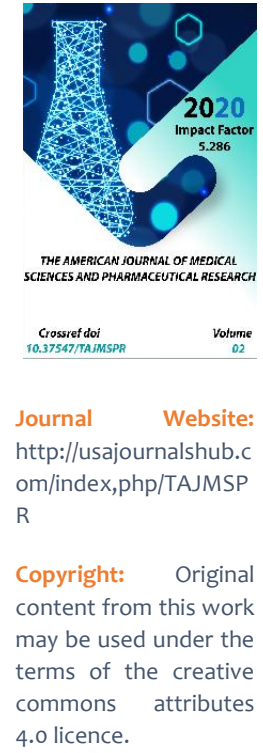

\title{
The Most Effective Drugs For Pecilomycose
}

Vahidova Adolat Mamatkulovna

Samarkand State Medical Institute, Uzbekistan

Hudoyarova Gavxar Nurmamatovna

Samarkand State Medical Institute, Uzbekistan

\section{ABSTRACT}

Echinococcosis remains a problematic issue in Uzbekistan as well. Many Uzbek and Russian scientists have been studying the spread of echinococcosis among the population and farm animals of Uzbekistan. In this article, we discuss of echinococcosis as the main issue in our country.

\section{KEYWORDS}

Echinococcosis, pesilomycosis, farm animals, population, spread.

\section{INTRODUCTION}

According to T. Abdiev et al. (2000), echinococcosis among the population and animals is widespread and tends to grow. According to M. Aminjanov (2004), the infestation of farm animals and dogs, as well as in humans, increases from year to year. And therefore, the problem of echinococcosis among animals and humans remains relevant. A completely new direction in the study of echinococcosis is being put forward. For example, before our research, there was not a single work devoted to the mixed course of echinococcosis with pecilomycosis. There were only separate studies of the publication by F. Nazyrov et al. (2004), which clearly shows the combination of parasitic echinococcus with fungi. However, the distribution of petsilomycosis among 
domestic animals, birds and rodents has not been studied at all, there was also not a single work devoted to determining the role of pesilomycosis on the course of echinococcosis. Serious electron microscopic studies of echinococcus with fungi have been carried out, but the species of fungi has not been established.

The distribution of petsilomycosis among domestic animals, rodents and birds has not been studied at all. Currently, a completely new direction is being put forward in the study of echinococcosis with petsilomycosis. For example, prior to our research, there was not a single work devoted to the study of the mixed course of echinococcosis and petsilomycosis, both among animals and in humans.

Pecilomycosis is a new type of systemic mycosis caused by infection with various types of fungi of the genus Paecilomyces, in which, along with damage to the skin and mucous membranes, deep, systemic lesions of various organs, as well as its septic forms, are described. In addition to humans, petsilomycosis affects birds, domestic and wild animals. The first report on human infection with a fungus of the genus Paecilomyces of the species Paecilomyces variotii Bainier (1907) was made by cardiac surgeons from South Africa (Uys et al., 1963). Over the past period, the pathogenic properties of several more species of fungi of this genus have been described, among which 3 species: P.variotii (Gazikhojaeva M.A., et al. 1978), P. carneus (Kornishcheva V.G., 1987) and P.viridis (Akhunova A.M., 1991) were isolated from patients living in Uzbekistan. Infection with pecilomycosis is not associated with visiting endemic areas and certain geographic latitudes.

Fungi of the genus Paecilomyces are ubiquitous in nature, which suggests a massive infection of the world's population with them. So, for example, in an epidemiological study for petsilomycosis among residents of the Samarkand region, we established a general infection of the population with fungi of the Paecilomyces genus, which has low virulence and weak antifoam properties, as evidenced by their long persistence in the blood of humans and animals without signs of an infectious process, and them into the group of conditionally pathogenic microorganisms.

\section{MATERIALS AND RESEARCH METHODS}

Methods of helminthological, morphological, hematological, bacteriological, biochemical, immunological and serological studies were used in the work.

\section{RESEARCH RESULTS AND THEIR DISCUSSION}

Until now, echinococcosis has been studied as an independent disease. None of the researchers paid attention to, or to the fact, what effect petsilomycosis has on the course of pulmonary echinococcosis. Therefore, in our studies, we tried to determine the role of the causative agent of petsilomycosis on the course of echinococcosis of the lungs of animals and humans.

The clinical material covers 104 patients operated on for echinococcosis in the clinic of the Department of Faculty and Hospital Surgery of the SamGosMI. In 104 patients with concomitant echinococcosis of the lungs and liver, the concentration of spherules of the fungus of the genus Paecilomyces was higher than 17000 in $1 \mu \mathrm{l}$ of blood.Analysis of the results of treatment of patients showed that the use of minimally invasive approaches and simultaneous operations of the lungs and 
liver contributed to a reduction in the treatment time of patients from $26.4 \pm 4$ days in the control group up to $14.6 \pm 3$ days in the main group. Thus, performing operations through minimally invasive approaches in combination with video assistance did not lead to an increase in the number of postoperative complications in comparison with operations performed by traditional thoracotomy and laparotomy approaches, which made it possible to significantly shorten the treatment time of patients, minimize the number of purulent postoperative complications and exclude such long-term complications, such as postoperative hernias, ligature fistulas, gross deforming scars and adhesions of the abdominal cavity, thereby shortening the treatment time of patients in the hospital, reducing the consumption of medicines in comparison with step-by-step treatment. There was a decrease in the time taken to perform the operative access and the total duration of the operation. Such patients with echinococcosis were previously treated by surgical clinicians with fungicides: diflazone, fluconazole, diflucan, orungal, homeopathic todicamp.

Our studies are also devoted to 87 patients with combined-multiple (lung and liver) echinococcosis, complicated by pecilomycosis. Served as control 12 apparently healthy individuals. All observed patients showed a violation of amino acid metabolism, which was characterized by an increase in the amount of individual free amino acids in the blood with a decrease in the content of their excretion in the urine and a decrease in the amount of other free amino acids in the blood with an increase in their excretion in the urine. Impaired immune status was characterized by a statistically significant $(P<0.01)$, compared with control, by a decrease in the number of
$\mathrm{CDZ}+, \mathrm{CD} 4+, \mathrm{CD} 8+, \mathrm{CD} 16+, \mathrm{CD} 21+$, phagocytosis, an increase in the number of immunoglobulins and circulating immune complexes. In all the patients we observed, the number of spherules of the fungus Paecilomyces was more than 19 thousand in 1 $\mu$ of blood compared to the norm of up to 6 thousand in $1 \mu$ in clinically healthy individuals. The first group - 7 patients were treated with Diflucan for two weeks, the second - 5 patients with severe drug allergy did not receive antifungal and immunomodulatory drugs, the third - for two weeks received the homeopathic drug Cheblin-SK-1, the fourth for two weeks polyoxidonium, the fifth - used homeopathic irillen for two weeks. Patients of the 6th group received mycosyst internally, the 7th group received mycosyst intravenously. There were 15 patients in groups 3-7. On the 4-5th day after oral administration of $150 \mathrm{mg}$ of mycosyst in a capsule for 10 days in two patients, nausea, vomiting, and convulsions were noted in two patients. The drug was discontinued and the patients were successfully treated with homeopathic irillen. In the group of patients who received mycosyst intravenously, there were no side and toxic effects of the drug. Therefore, mycosyst capsules should be administered with caution. After the treatment in the blood of patients of the 1st and 5 th groups, the number of spherules in the blood returned to normal and fluctuated within the range of 3-6 thousand in $1 \mu$ l. However, there was no normalization of indicators of amino acid metabolism and immune status.

6 months after the repeated course of treatment, a tendency towards normalization of amino acid metabolism and immune status was observed in patients from groups 3,4,5. One year after the course of treatment, amino 
acid metabolism and immune status returned to normal in patients of groups 3-5. All patients in the course of treatment were successfully operated using modern technologies for the surgical treatment of echinococcosis.

In order to suppress pecilomycosis infection in the postoperative period, 15 patients used nizoral for 14 days, 15 patients - diflucan, 15 patients - nystatin, 44 children received homeopathic irillen for 2 weeks, 1 crumble per day. 16 children were treated with kefadin + mycosyst. Intravenous administration of Mycosyst had no side or toxic effects, but the capsules had side effects. After the course of treatment, the blood was tested again for the content of Petsilomyces. No side and toxic effects of the drugs used have been established. Nystatin did not provide a decrease in the level of pecilomyces in the blood of sick children. In 12 children, nizoral provided a decrease in the number of spherules of petsilomyceses to 3-4 thousand in $1 \mu \mathrm{l}$ of blood, which is considered the norm. Diflucan, mycosyst and homeopathic irillen resulted in normal concentration of fungus spherules in the blood of treated children. It should also be borne in mind that fungi of the genus Paecilomyces are conditionally pathogenic and are found in all children who have had echinococcosis.

After the antifungal treatment with Diflucan, the number of fungal spherules in the blood returned to normal in 12 children, in 10 children with nizoral, and in 15 children with todicamp. The children were successfully operated on with the removal of echinococcal cysts. Three months after the operation, the children were repeated the course of antifungal treatment. A year after surgery and a course of antifungal treatment, the children recovered their immune status and amino acid metabolism.

\section{CONCLUSION}

1. Echinococcosis proceeds against the background of pecilomycosis, which in itself causes a sharp violation of the immune system. At this time, in the blood of patients with echinococcosis complicated by pecilomyccosis, the number of fungal spherules exceeds 17 thousand in $1 \mu \mathrm{l}$. The treatment of such patients for 10 days, including antifungicidal drugs, and homeopathic ones, leads to a decrease in the number of spherules in the blood of the treated patients to 5-6 thousand in $1 \mu \mathrm{l}$. A tendency to restore the immune status begins to appear. Effective treatment of echinococcosis complicated by petsilomycosis is confirmed by published works.

2. Based on the studies and observations, analysis and discussion of the results of the study, we come to the conclusion that antifungicidal drugs, together with homeopathic drugs, contribute to a speedy recovery, which in turn have, in addition to them, antiechinococcosis and anti-cyclomycotic qualities, as well as hepatoprotective, immunotropic and analgesic properties.

\section{REFERENCES}

1. Askarov K.A., Abdullaev N., Sabirov B.U., Prokina E.S. Pathogenetic therapy in the surgical treatment of echinococcosis using homeopathic todicamp // Problems of ecology, health, pharmacy and parasitology. - M., 2005. - pp. 56-61. 
2. Ashurov A.A., Zakirova N.I., Prokina E.S., Askarov K.A. Experimental pecilomycosis caused by infection of animals with material from patients with bronchial asthma and echinococcosis complicated by pecilomycosis // Proceedings of the 1st Congress of CIS Physiologists. Physiology and human health. - M., 2005. - T.2. - S. $184-185$.

3. Ganizoda H.G., Chebyshev N.V., Malenkov A.G. et al. Amino acid metabolism in echinococcosis and other liver diseases and its normalization with the help of todicamp and CSK-1 preparations // Echinococcosis of the abdominal organs and rare localizations. - M., 2004 .-- S. 183 .- 188.

4. Ahmadi N.A. Using morphometry of the larval rostellar hooks to distinguish Iranian strains of Echinococcus granulosus // Annals of Tropical Medicine and Parasitology. - 2004 - Vol.98. - No. 3. - P. 211-220 (10)

5. Burgu A., Sarimehmetoglu O., Vural S. A. Cystic echinococcosis in a stray cat // The Veterinary Record. - 2004 -Vol. 155. - No. 22.27. - P. 711-712 (2)

6. Dadaev Sh.A., Ashurmetov A.M., Eshov R.U. et al. Endolymphatic antibioticotherapy after echinococcectomy // Int. symposium. Surgery of echinococcosis. - Khiva, 1994. P.21-22.

7. Karimov Sh.I. Problem of echinococcosis in Uzbekistan - achienements and Perspectives // Int. symposium. Surgery of echinococcosis. - Khiva, 1994. - P. 1-3.

8. Swiderski Zdzislaw, Malczewski Andrzej. WHO / OIE Manual on Echinococcosis in Humans and Animals: a Public Health Problem of Global Concern // Acta Parasitologica. - 2002- Vol.47 - No. 2. P.177
9. Veraalvarez J. et al. Echnococcus hookjets in sputum sinus trast extension of hydratid cyst to lung // Acta Cytalogica. -1995. №6. - P. 39. 